Kazuhiko Yamada Chizuko Nishida-Umehara •

Yoichi Matsuda

\title{
A new family of satellite DNA sequences as a major component of centromeric heterochromatin in owls (Strigiformes)
}

Received: 30 March 2004 / Accepted: 30 March 2004 / Published online: 16 April 2004

C) Springer-Verlag 2004

\section{Chromosoma (2004) 112:277-287}

In the pdf version online and in the print version, Fig. 9 was a duplication of Fig. 5. The correct Fig. 9 is given below.

The online version of the original article can be found at http://dx. doi.org/10.1007/s00412-003-0267-z

K. Yamada · C. Nishida-Umehara · Y. Matsuda

Laboratory of Cytogenetics, Division of Bioscience, Graduate School of Environmental Earth Science, Hokkaido University, Japan

K. Yamada $\cdot$ C. Nishida-Umehara $\cdot$ Y. Matsuda $(\triangle)$ Laboratory of Animal Cytogenetics, Center for Advanced Science and Technology, Hokkaido University,

North10 West 8, Kita-ku,

Sapporo 060-0810, Japan

e-mail: yoimatsu@ees.hokudai.ac.jp

C. Nishida-Umehara · Y. Matsuda

Chromosome Research Unit, Faculty of Science, Hokkaido

University,

Sapporo, Japan

\begin{tabular}{|c|c|c|c|c|c|c|c|}
\hline & & 10 & 20 & 30 & 40 & 50 & \\
\hline & & CCAAGTGTTC & ACACITITITT & TC--- & & -TCCG-- & \\
\hline BU11 & (AB103207) & & & ...-... & & - A. - - & \\
\hline$J 16$ & (AB103208) & & & c. --. & & & \\
\hline 118 & & & c & G---- & G. & $-\ldots A-$ & \\
\hline 331 & (AB103210) & & & - & & $--\ldots$ & \\
\hline U36 & (AB1032 & & .... A. . & ...- & & $--\ldots$ & \\
\hline & & & $\ldots \mathrm{T} \ldots \ldots \mathrm{C}$ & ...... & .. T. . & -... T. - & T. \\
\hline 13 & (AB103213) & & $\ldots \mathrm{T} \ldots \mathrm{A}$ & G- & & -.. . Т. - - & \\
\hline 14 & (AB103 & & $\ldots \mathrm{T}$. & T- & & $--\ldots \ldots$ & \\
\hline & & & $\ldots \ldots \ldots c$ & & & $--\ldots .--$ & \\
\hline 19 & (AB10 & & $\ldots \ldots \ldots c$ & & .. T. . & $--\ldots .--$ & \\
\hline 110 & (AB1 & & . A & & С..... Т. & $--\ldots$ & \\
\hline 12 & $\mathrm{AB}$ & & $\ldots \mathrm{T} \ldots \ldots \mathrm{A}$ & & _... А. Т. Т. & $--\ldots \ldots$ & \\
\hline 37 & & . A. & $\ldots$ T.G..C & & & --. Т. & \\
\hline iL42 & $(\mathrm{AB} 1$ & & $\ldots T \ldots \ldots$ & G- & A. . & $-\ldots$ T. & \\
\hline-48 & (AB10 & & $\ldots$ Т. С... & G- & & $--\ldots$. & \\
\hline 51 & $(t$ & & $\ldots$ T.G..G & & & $-\ldots . . .-$ & \\
\hline 3L54 & (AB1 & & $\ldots T \ldots \ldots$ & G- & & -A. Т. - & \\
\hline s1 & (AB10 & . AC. . & $\ldots \mathrm{T} \ldots \mathrm{GCC}$ & G- & А. .... A ... & TT. TTCCGCG & A. A. A... \\
\hline 55 & &.$A C$ & $\ldots \mathrm{T} \ldots \mathrm{GCC}$ & & A. $\ldots$ A. . & CCGCA & c.... \\
\hline s6 & &.$A C$ & $\ldots \mathrm{T} \ldots \mathrm{GC}$ & & А.... T. Т. . & CGCG & c.. \\
\hline S7 & $(A B 1$ &.$A C$ & ...T. . GCC & G- & A. . A . . & -CCGCA & . C... \\
\hline$\$ 10$ & (AB1 & . AC. & $\ldots$. . . GCC & & А. $\ldots$ A. . & -AGGCA & A. A. C... \\
\hline S4 & & & & G- & G. T... & -- . T. - & \\
\hline S7 & (AB10 & & $\ldots \ldots \ldots c$ & G-- A. & & $-\ldots A-$ & . T...G \\
\hline is9 & (AB1 & & $\ldots \ldots \ldots c$ & & & $--\ldots$. & $\ldots . G$ \\
\hline$\$ 12$ & & A. . & $\ldots$. T. . C.G & & T. T. . &.- & \\
\hline 13 & (ABIC & & $\ldots \ldots \ldots c$ & $G-$ & & - - T. - & \\
\hline$S 15$ & (AB10 & & $\ldots \mathrm{T} \ldots \mathrm{A}$ & & _. A. Т. Т. . & $--\ldots$. & T. . \\
\hline C1 & & AC. & $\ldots \mathrm{T} \ldots \mathrm{GCC}$ & & A. ... A. C. . & TT. TTCCGCA & . C... \\
\hline & & $A C$. & $\ldots \mathrm{T} \ldots \mathrm{GCC}$ & & A. $\ldots$ A $\ldots$ & T-CCGCA & C... \\
\hline C5 & (AB1 1 & . AC. & $\ldots \mathrm{T} \ldots \mathrm{GCC}$ & & A. . A $\ldots$ & TT. T-CCGCA & A. A. . C... \\
\hline $\mathrm{C} 11$ & (AB1 & $\therefore \mathrm{AC}$ & $\ldots \mathrm{T} \ldots \mathrm{GCC}$ & G-_- & A. ... A. . & -CCGCA & A. A. C. . \\
\hline & & AG..... & $\ldots$ Т...... & . T-- - G. & $\ldots \ldots$ A. & GGCGT. GCCG & . A. \\
\hline PE9 & (AB103 & & . . $\mathrm{TT}$ & T-O-G. & $\ldots$..... & GGCGT. GCCG & . A. . \\
\hline PE14 & $(A B 1$ & & $\ldots \mathrm{T}$. & . . TाTCC. & . A. . & GG. GT. TCCG & A. . \\
\hline E16 & & & .. TT. & . TTT-G. & . A. & GGCGT. GCCG & A. . \\
\hline SUH1 & (AB103 & AG. & ... T. GC. - - &..-- & . T. . & --. GT. --- & $\ldots$ T. AA . A A \\
\hline UH4 & $\left(\mathrm{AB} 10_{3}\right.$ & & ... T. GC. . - & -. - & & -AGT.--- & $\ldots A A \ldots G$ \\
\hline H6 & & $\ldots$ & ... T. GC. - & $-\ldots$ & ..... T. . & -- GTA--- & $\ldots A A \ldots G$ \\
\hline 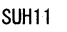 & & & & & & & \\
\hline
\end{tabular}

Fig. 9 Nucleotide sequences of the 41 monomeric fragments of the repetitive sequences cloned from Hae III-digested genomic DNA of B. bubo (BBU), Bubo virginianus(BVI), K. blakistoni (KBL), Ny. scandiaca (NYS), Otus scops (OSC) and P. perspicillata(PPE), and from Eco RI-digested genomic DNA of $S$. u. hondoensis (SUH) and the Hin fI-digested genomic DNA of Ni. scutulata (NIS) 


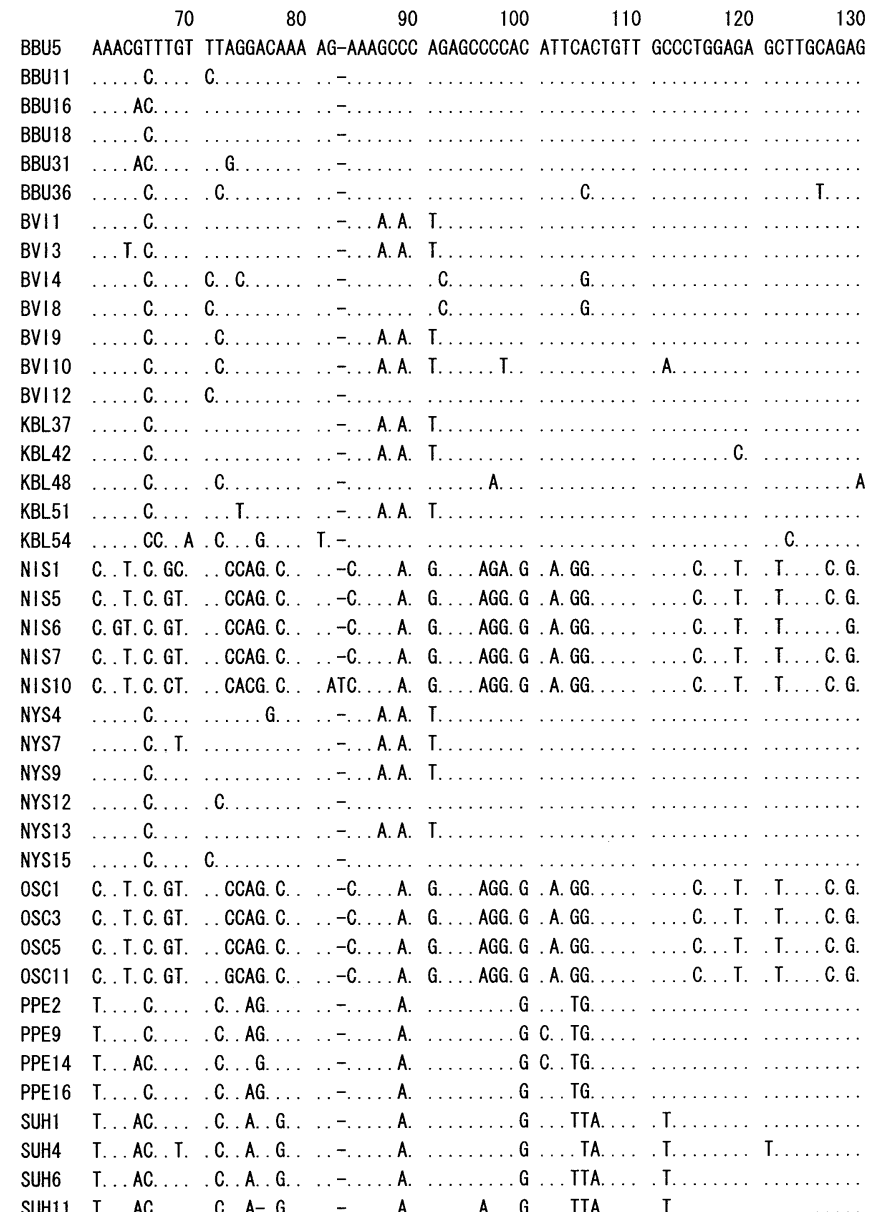

Fig. 9 (continued)

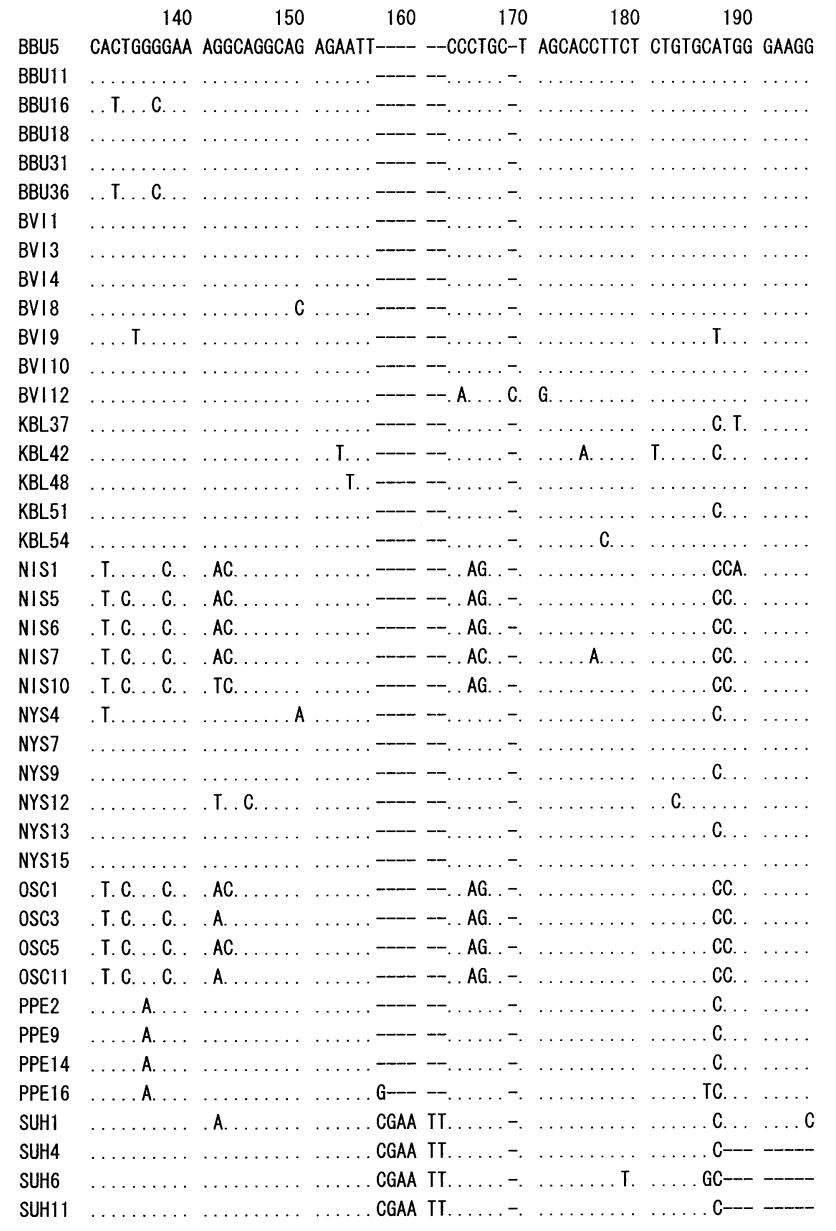

Fig. 9 (continued) 